Pacific Journal of Mathematics

THE COMMON FIXED POINT THEORY OF SINGLEVALUED 


\title{
THE COMMON FIXED POINT THEORY OF SINGLEVALUED MAPPINGS AND MULTIVALUED MAPPINGS
}

\section{SHIGERU ITOH AND WATARU TAKAHASHI}

\begin{abstract}
First, in a locally convex topological vector space, a theorem is proved which extends fixed point theorems by Lau and Fan-Glicksberg. In a strictly convex Banach space, a similar result is obtained, which is a generalization of the fixed point theorem by Bohnenblust-Karlin. In a Banach space which satisfies Opial's condition, a fixed point theorem is given that generalizes both results by Holmes-Lau-Lim and Lami Dozo. In a uniformly convex Banach space, a similar theorem is considered which extends Lim's fixed point theorem. Finally, the existence of common fixed points of a quasi-nonexpansive mapping and a multivalued nonexpansive mapping is established by an elementary constructive method in a Hilbert space. In many cases, preliminary results on nonexpansive or quasi-nonexpansive retractions are obtained which play crucial roles in proving the above theorems.
\end{abstract}

1. Introduction. De Marr [11] proved that if $G$ is a commutative family of nonexpansive mappings on a compact convex subset $K$ of Banach space, then $G$ has a common fixed point in $K$. Then results for nonexpansive mappings on weakly compact convex subsets appeared. Browder [5] proved a fixed point theorem for a single nonexpansive mapping on a bounded closed convex subset of a Hilbert space, while Browder [6] and Göhde [19] on a bounded closed convex subset of a uniformly convex Banach space. Kirk [23] obtained a general form of the similar result for a single nonexpansive mapping on a weakly compact convex subset $K$ of a Banach space in the case that $K$ has normal structure. Since then, various fixed point theorems for nonexpansive mappings were given by Belluce and Kirk [2, 3], Takahashi [33, 34], Mitchell [31], Kirk [24], Holmes and Lau [21], Dotson [12], Lau [26], Bruck [9, 10] and Lim [27], etc. Among them, Bruck obtained interesting characterizations of fixed point sets of nonexpansive mappings. There were also Dotson's results [13] on fixed points of quasi-nonexpansive mappings. Lim [28] proved that if $K$ is a weakly compact convex subset of a Banach space and $K$ has normal structure, then $K$ has complete normal structure. Hence combining this with a theorem of Holmes and Lau [21], it follows that if $G$ is a left reversible semigroup of nonexpansive mappings on a weakly compact convex subset $K$ of a 
Banach space and $K$ has normal structure, then there is a common fixed point of $G$ in $K$.

On the other hand, fixed point theorems for multivalued upper semicontinuous mappings were proved by Bohnenblust and Karlin [4], Glicksberg [17], Fan [15], Browder [8], and Takahashi [35], etc. And fixed point theorems for multivalued nonexpansive mappings were given by Markin [32], Lami Dozo [25], Assad and Kirk [1] and Lim [29], etc. Contrary to singlevalued cases, results for multivalued nonexpansive mappings on weakly compact convex subsets (which have normal structure) of general Banach spaces are not yet obtained up to the present.

In [22] we examined the existence of common fixed points for a singlevalued mapping and a multivalued mapping. In this paper we give various common fixed point theorems for families of singlevalued nonexpansive or quasi-nonexpansive mappings and multivalued upper semicontinuous or nonexpansive mappings. These generalize both results in singlevalued and multivalued cases simultaneously. We also obtain some theorems on nonexpansive or quasi-nonexpansive retractions. At first, in $\S 3$ we prove theorems for semigroups of nonexpansive mappings and multivalued upper semicontinuous mappings in locally convex topological vector spaces, while in $\S 4$ those for families of nonexpansive or quasi-nonexpansive mappings and multivalued nonexpansive mappings in Banach spaces. Finally, in $\S 5$ we give more precise results in Hilbert spaces.

The authors wish to express their gratitude to Professor $\mathrm{H}$. Umegaki for many suggestions and advices in preparing this paper.

2. Preliminaries. Let $X$ be a topological space, $2^{X}$ the family of all subsets of $X, T$ a mapping of $X$ into $2^{X}$ such that $T x$ is nonempty for all $x \in X . \quad T$ is called upper semicontinuous if for each closed subset $C$ of $X, T^{-1}(C)=\{x \in X: T x \cap C \neq \varnothing\}$ is closed.

Let $E$ be a locally convex topological vector space, $Q$ a family of continuous seminorms that generates the topology of $E$. Let $X$ be a nonempty subset of $E, f$ a mapping of $X$ into $E$. Denote by $F(f)$ (which may be empty) the set of fixed points of $f$ in $X . \quad f$ is called Q-nonexpansive with respect to $M$ (a nonempty subset of $X$ ) if for any $p \in Q, p(f x-f u) \leqq p(x-u)$ whenever $x \in X$ and $u \in M$. If $M=X$, then $f$ is called $Q$-nonexpansive, and if $M=F(f)$, then $f$ is called $Q$-quasi-nonexpansive respectively.

Let $B$ be a Banach space, $X$ a nonempty subset of $B, f$ a mapping of $X$ into $B . f$ is said to be k-contraction (where $0 \leqq k<1$ ) if for any $x, y \in X,\|f x-f y\| \leqq k\|x-y\| . \quad f$ is said to be nonexpansive with respect to $M$ (a nonempty subset of $X$ ) if for each $x \in X$, $u \in M$, $\|f x-f u\| \leqq\|x-u\|$. If $M=X, f$ is said to be nonexpan- 
sive, and if $M=F(f), f$ is said to be quasi-nonexpansive. $f$ is said to be generalized nonexpansive if there exist nonnegative real numbers $a, b, c$ with $a+2 b+2 c \leqq 1$ such that for any $x, y \in X$,

$$
\begin{aligned}
\|f x-f y\| & \leqq a\|x-y\|+b\{\|x-f x\|+\|y-f y\|\} \\
& +c\{\|x-f y\|+\|y-f x\|\} .
\end{aligned}
$$

Note that if a generalized nonexpansive mapping has fixed points, then it is quasi-nonexpansive. Let $T$ be a mapping of $X$ into $2^{X}$ such that for each $x \in X, T x$ is nonempty bounded closed and let $D$ be the Hausdorff metric on nonempty bounded closed subsets of $B$ induced by the norm of $B$. If for any $x, y \in X, D(T x, T y) \leqq\|x-y\|$, $T$ is called nonexpansive.

Let $G$ be a semitopological semigroup, that is, $G$ is a semigroup with a Hausdorff topology such that the semigroup operation $G \times G \rightarrow G$ by $(s, t) \rightarrow s t(s, t \in G)$ is separately continuous. $G$ is said to be left reversible if any two nonempty closed right ideals of $G$ have nonvoid intersection. Let $C(G)$ be the Banach algebra of all continuous bounded realvalued functions on $G$ with sup norm. For each $t \in G$, define the operators $r_{t}, l_{t}$ on $C(G)$ by $\left(r_{t} h\right)(s)=h(s t)$, $\left(l_{t} h\right)(s)=h(t s)$ for all $s \in G$ and $h \in C(G)$. Let $A$ be a subspace of $C(G)$ containing the constant function 1 . An element $m$ of the dual space $A^{*}$ of $A$ is called a mean if $m(1)=\|m\|=1$. For any $h \in C(G)$, denote $r_{G} h=\left\{r_{t} h: t \in G\right\}$. Then $A P(G)=\left\{h \in C(G): r_{G} h\right.$ is precompact in $C(G)\}$ is a left and right translation invariant (i.e., $l_{t}(A P(G)) \subset$ $A P(G), \quad r_{t}(A P(G)) \subset A P(G)$ for all $\left.t \in G\right)$ closed subalgebra of $C(G)$ containing 1. A mean $m$ on $A P(G)$ is called a left invariant mean if $m\left(l_{t} h\right)=m(h)$ for all $h \in A P(G)$ and $t \in G$. If $G$ is left reversible, then $A P(G)$ has a left invariant mean (cf. $[21,26]$ ).

An action of a semitopological semigroup $G$ on a topological space $X$ is a mapping $G \times X \rightarrow X$ such that $(s t) x=s(t x)$ for all $s, t \in G$ and $x \in X$, where $t x$ denotes the image of $(t, x)$. The action is called separately continuous if the mapping $G \times X \rightarrow X$ is separately continuous. If $X$ is a subset of a Banach space $B$ (a locally convex space $E$ ), then the action of $G$ on $X$ is called $(Q-)$ nonexpansive if for each $s \in G$, the mapping of $X$ into $X$ defined by $x \rightarrow s x(x \in X)$ is $(Q-)$ nonexpansive.

Let $X$ be a nonempty subset of a locally convex topological vector space $E$ (or a Banach space $B$ ), $f$ a mapping of $X$ into $X, G$ a family of mappings of $X$ into $X, T$ a mapping of $X$ into $2^{X}$ such that $T x$ is nonempty for every $x \in X$. We denote by $G x$ the set $\{g x: g \in G\}$ for any $x \in X$, and by $F(G)$ (which may be nonempty) the set of common fixed points of $G$ in $X$. Let $Y$ be a empty subset of $X$, then we denote by $b d_{X} Y$ the relative boundary of $Y$ with 
respect to $X$, that is, $b d_{X} Y=\mathrm{cl}(Y) \cap \mathrm{cl}(X \backslash Y)$, where $\mathrm{cl}(Z)$ is the closure of $Z . \quad Y$ is said to be $f$-invariant if $f(Y) \subset Y . \quad Y$ is said to be $G$-invariant if $Y$ is $g$-invariant for all $g \in G$. $f$ and $T$ is said to commute if for each $x \in X, f(T x) \subset T(f x) . \quad f$ and $T$ is said to commute weakly if for any $x \in X, f\left(b d_{X} T x\right) \subset T(f x) . \quad G$ and $T$ is said to commute (weakly) if each $g \in G$ and $T$ commute (weakly). Let $G$ be a semitopological semigroup acting on $X$, then we also denote by $F(G)$ the set of fixed points of the semigroup of mappings $J=$ $\{x \rightarrow s x(x \in X): s \in G\} . \quad G$ and $T$ is said to commute (weakly) if $J$ and $T$ commute (weakly). $Y$ is called $G$-invariant if $Y$ is $J$-invariant. A mapping $r$ of $X$ into $X$ is called a retraction if $r^{2}=r$.

Now we give some results in Banach spaces.

Proposition 1. Let $K$ be a nonempty closed convex subset of a Banach space $B, f$ a mapping of $K$ into $B$ such that $M=\{y \in K$ : $\|f y-y\|=\min \{\|f y-x\|: x \in K\}\}$ is nonempty and $f$ is nonexpansive with respect to $M$. Then $M$ is a closed set on which $f$ is continuous. Furthermore, if $B$ is strictly convex and $f$ is isometric on $M$, then $w=k u+(1-k) v(u, v \in M, 0<k<1)$ implies that fw $=$ $k f u+(1-k) f v$.

Proof. It is obvious that $f$ is continuous at each point of $M$. We show that $M$ is closed. Let $\left\{y_{n}\right\}$ be a sequence of $M$ which converges to $z \in K$. For any $c>0$, take $m=m(c)$ such that $\left\|y_{m}-z\right\|<c$, then

$$
\begin{aligned}
\|f z-z\| & \leqq\left\|f z-f y_{m}\right\|+\left\|f y_{m}-y_{m}\right\|+\left\|y_{m}-z\right\| \\
& \leqq\left\|y_{m}-z\right\|+\left\|f y_{m}-y_{m}\right\|+\left\|y_{m}-z\right\| \\
& <2 c+\left\|f y_{m}-y_{m}\right\| .
\end{aligned}
$$

For each $x \in K$,

hence

$$
\begin{aligned}
\left\|f y_{m}-x\right\| & \leqq\left\|f y_{m}-f z\right\|+\|f z-x\| \\
& \leqq\left\|y_{m}-z\right\|+\|f z-x\| \\
& <c+\|f z-x\|
\end{aligned}
$$

$$
\begin{aligned}
\left\|f y_{m}-y_{m}\right\| & =\min \left\{\left\|f y_{m}-x\right\|: x \in K\right\} \\
& \leqq c+\inf \{\|f z-x\|: x \in K\} .
\end{aligned}
$$

Therefore, it follows that

$$
\|f z-z\|<3 c+\inf \{\|f z-x\|: x \in K\} .
$$

Since $c$ is arbitrary, we have $\|f z-z\| \leqq \inf \left\{\left\|f_{z}-x\right\|: x \in K\right\}$ and $z \in M$. Thus $M$ is closed.

Suppose $B$ is strictly convex and $f$ is isometric on $M$. Let 
$u, v \in M, 0<k<1$ and $w=k u+(1-k) v$. By assumption

$$
\|u-v\|=\|f u-f v\| \text { and }\|f w-f v\| \leqq\|w-v\| \text {. }
$$

Thus

$$
\begin{aligned}
\|u-w\| & =\|u-v\|-\|w-v\| \\
& \leqq\|f u-f v\|-\|f w-f v\| \\
& \leqq\|f u-f w\| \leqq\|u-w\| .
\end{aligned}
$$

Hence $\|f u-f w\|=\|u-w\|$. Similarly we have $\|f v-f w\|=$ $\|v-w\|$. Therefore, we obtain

$$
\begin{aligned}
\|f u-f w\|+\|f w-f v\| & =\|u-w\|+\|w-v\| \\
& =\|u-v\|=\|f u-f v\| .
\end{aligned}
$$

Since $B$ is strictly convex, it follows that $f w=k f u+(1-k) f v$.

For nonexpansive mappings a similar result to the second part of Proposition 1 was given by Edelstein [14]. As a direct consequence of Proposition 1, we have the following result which was due to Dotson [13].

COROLlaRY 1. Let $K$ be a closed convex subset of a strictly convex Banach space, $f$ a quasi-nonexpansive mapping of $K$ into $K$. Then $F(f)$ is a nonempty closed convex set on which $f$ is continuous.

Proof. Since $f(K) \subset K, F(f)$ equals the set $M$ as in Proposition 1. Thus, for any $u, v \in F(f), 0<k<1$ with $w=k u+(1-k) v$, we have $f w=k f u+(1-k) f v=k u+(1-k) v=w$. This implies that $F(f)$ is convex.

The following was given by Bruck [10].

Proposition 2. Let $X$ be a Hausdorff topological space, $S$ a semigroup of mappings of $X$ into $X$. If $S$ is compact in the topology of pointwise convergence on $X$ and for each $x \in X$, there exists a common fixed point of $S$ in $S x$, then there is in $S$ a retraction of $X$ onto $F(S)$.

3. Fixed point theorems in topological vector spaces. Throughout this section, let $K$ be a nonempty compact convex subset of a locally convex Hausdorff topological vector space $E, Q$ a family of continuous seminorms that generates the topology of $E$. The notion of nonexpansiveness always means $Q$-nonexpansiveness and we omit the term $Q$. 
THEOREM 1. Let $G$ be a family of mappings of $K$ into $K$ such that $F(G)$ is nonempty and each $g \in G$ is nonexpansive with respect to $F(G)$. If any $G$-invariant closed convex subset of $K$ has a common fixed point of $G$, then there exists a quasi-nonexpansive retraction $r$ of $K$ onto $F(G)$ for which every G-invariant closed convex subset of $K$ is r-invariant.

Proof. Define $S=\{s: K \rightarrow K: s$ is nonexpansive with respect to $F(G), F(s) \supset F(G)$ and any $G$-invariant closed convex subset of $K$ is $s$-invariant\}. Then $G \subset S$. We show that $S$ is compact in the topology of pointwise convergence. Fix $v \in F(G)$. For each $x \in K$, put

$$
W x=\{y \in K: p(y-v) \leqq p(x-v) \text { for all } p \in Q\},
$$

then $W x$ is nonempty compact convex. In fact, for any $s \in S$, $p(s x-v) \leqq p(x-v)$, hence $s x \in W x . \quad S$ can be regarded as a subset of the product topological space $W=\prod_{x \in K} W x$. Since $W$ is compact and the topology on $W$ is that of pointwise convergence, it suffices to show that $S$ is closed in $W$. Suppose that $\left\{s_{i}\right\}$ is a net in $S$ which converges to $s$ in $W$. For any $x \in K, u \in F(G), p \in Q$, we have

$$
p(s x-u)=\lim _{i} p\left(s_{i} x-u\right) \leqq p(x-u)
$$

and

$$
s u=\lim _{i} s_{i} u=u .
$$

It is obvious that if $C$ is a $G$-invariant closed convex subset of $K$, then $s(C) \subset C$. Hence $s \in S$ and $S$ is closed in $W$. It can be seen that $S$ is a semigroup and for any $s, t \in S, k(0 \leqq k \leqq 1), k s+(1-k) t \in S$. This implies that for each $x \in K, S x$ is compact convex and $G$-invariant. By assumption $S x$ has a common fixed point of $G$, that is, $S x$ has a common fixed point of $S$. Therefore, by Proposition 2 there exists in $S$ a retraction $r$ of $K$ onto $F(S)=F(G)$. Since $F(r)=F(G), r$ is quasi-nonexpansive.

CoROLlaRY 2. Let $f$ be a continuous quasi-nonexpansive mapping of $K$ into $K$, then there exists a quasi-nonexpansive retraction $r$ of $K$ onto $F(f)$ such that each $f$-invariant closed convex subset of $K$ is r-invariant.

Proof. Since $f$ is continuous, any $f$-invariant closed convex subset of $K$ has a fixed point of $f$ by Tychonoff's fixed point theorem.

For a nonexpansive action of a semitopological semigroup on $K$, we obtain the following. 
THEOREM 2. Let $G$ be a semitopological semigroup acting on $K$ such that $A P(G)$ has a left invariant mean. If the action of $G$ on $K$ is separately continuous and nonexpansive, then there exists a nonexpansive retraction $r$ of $K$ onto $F(G)$ such that every $G$-invariant closed convex subset of $K$ is r-invariant.

Proof. $F(G)$ is nonempty by Lau's theorem [26]. Put $S=$ $\{f: K \rightarrow K: f$ is nonexpansive with $F(f) \supset F(G)$ and any $G$-invariant closed convex subset of $K$ is $f$-invariant\}, then as in the proof of Theorem $1, S$ is a semigroup and compact in the topology of pointwise convergence. For each $x \in K, S x$ is a $G$-invariant compact convex subset of $K$, so by [26] again there exists a common fixed point of $G$ in $S x$ which is also a fixed point of $S$. Thus, by Proposition 2 there is in $S$ a retraction $r$ of $K$ onto $F(S)=F(G)$.

Now we give a common fixed point theorem for a semigroup of nonexpansive mappings and a multivalued upper semicontinuous mapping.

THEOREM 3. Let $G$ be a semitopological semigroup acting on $K$ for which $A P(G)$ has a left invariant mean. Suppose the action of $G$ on $K$ is separately continuous and nonexpansive. Let $T$ be an upper semicontinuous mapping of $K$ into $2^{K}$ such that for each $x \in K, T x$ is nonempty compact convex. If $G$ and $T$ commute, then there exists an element $z \in K$ such that $g z=z \in T z$ for all $g \in G$.

Proof. By Theorem 2 there exists a nonexpansive retraction $r$ of $K$ onto $F(G)$ for which every $G$-invariant closed convex subset of $K$ is $r$-invariant. Define a mapping $S$ of $K$ into $2^{K}$ by $S x=T(r x)$ $(x \in K)$, then $S$ is upper semicontinuous. By Fan-Glicksberg's fixed point theorem [15], [17] there is a $v \in K$ such that $v \in S v$. Since for any $g \in G, g(S v)=g(T(r v)) \subset T(r v)=S v$, it follows that $r(S v) \subset S v$ and in particular $r v \in S v$. Put $z=r v$, then we have $g z=z \in T z$ for all $g \in G$.

If $G$ is generated by a single mapping and $T$ is a singlevalued mapping, then the following holds.

CoROLlary 3. Let $f$ be a continuous mapping of $K$ into $K, g$ a nonexpansive mapping of $K$ into $K$. If $f$ and $g$ commute, then there exists $a z \in K$ such that $f z=g z=z$.

4. Fixed point theorems in Banach spaces. In this section we consider various common fixed point theorems for singlevalued 
mappings and multivalued mappings in Banach spaces. At first, we have the following theorem for a family of quasi-nonexpansive mappings and an upper semicontinuous multivalued mapping on a compact convex subset of a strictly convex Banach space.

THEOREM 4. Let $K$ be a nonempty compact convex subset of a strictly convex Banach space, $G$ a family of mappings of $K$ into $K$ for which $F(G)$ is nonempty and every $g \in G$ is nonexpansive with respect to $F(G), T$ ian upper semicontinuous mapping of $K$ into $2^{K}$ such that for each $x \in K, T x$ is nonempty closed convex. If $G$ and $T$ commute weakly, then there exists a point $z \in K$ such that $g z=z \in T z$ for all $g \in G$.

Proof. By Corollary $1 F(G)$ is closed convex. Choose any point $u \in F(G)$. For each $x \in F(G), T x$ is nonempty closed convex, hence there exists a unique element $v \in b d_{K} T x$ nearest to $u$. Since for any $g \in G, g\left(b d_{K} T x\right) \subset T(g x)=T x$ and $g$ is nonexpansive with respect to $F(G)$, it follows that $\|g v-u\| \leqq\|v-u\|$ and $g v=v$. Thus we have $T x \cap F(G) \neq \varnothing$ for all $x \in F(G)$. Now define a multivalued mapping $S$ of $F(G)$ into $2^{F(G)}$ by $S x=T x \cap F(G)(x \in F(G))$. Then it is obvious that $S$ is upper semicontinuous. By the fixed point theorem of Bohnenblust and Karlin [4], we obtain a point $z \in F(G)$ such that $z \in S z$. Hence $g z=z \in T z$ for all $g \in G$.

CoRollary 4. Let $K$ be a nonempty compact convex subset of a strictly convex Banach space, $f$ a continuous generalized nonexpansive mapping of $K$ into $K, T$ an upper semicontinuous mapping of $K$ into $2^{K}$ such that for any $x \in K, T x$ is nonempty closed convex. If $f$ and $T$ commute weakly, then there exists an element $z \in K$ for which $f z=z \in T z$.

Proof. By Schauder's fixed point theorem, $F(f)$ is nonempty, thus $f$ is quasi-nonexpansive.

On a weakly compact convex subset of a strictly convex Banach space, an analogous result to Theorem 1 holds without any assumption.

THEOREM 5. Let $K$ be a nonempty weakly compact convex subset of a strictly convex Banach space, $G$ a family of mappings of $K$ into $K$ for which $F(G)$ is nonempty and any $g \in G$ is nonexpansive with respect to $F(G)$. Then there exists a quasi-nonexpansive retraction $r$ of $K$ onto $F(G)$ such that each G-invariant closed convex subset of $K$ is r-invariant. 
Proof. We make use of methods employed by Bruck [9, 10]. Put $S=\{s: K \rightarrow K: s$ is nonexpansive with respect to $F(G), F(s) \supset$ $F(G)$ and every $G$-invariant closed convex subset of $K$ is $s$-invariant\}. Then $G \subset S$. It is obvious that $S$ is a semigroup of mappings of $K$ into $K$. We show that $S$ is compact in the topology of pointwise weak convergence on $K$. Fix an element $v \in F(G)$. For each $x \in K$, denote $W x=\{y \in K:\|y-v\| \leqq\|x-v\|\}$. Then since for any $s \in S$, $\|s x-v\| \leqq\|x-v\|, S x \subset W x$ and $W x$ is closed convex. Since $K$ is weakly compact, $W x$ is weakly compact. $S$ is a subset of the product topological space $W=\Pi_{x \in K} W x$ (each $W x$ is endowed with the weak topology). $W$ is compact and the topology of $W$ is that of pointwise weak convergence, hence it is sufficient to prove that $S$ is closed in $W$. Let $\left\{s_{i}\right\}$ be a net in $S$ which converges to $s$ in $W$, then for any $x \in K, u \in F(G)$,

$$
\begin{aligned}
\|s x-u\| & =\left\|w-\lim _{i}\left(s_{i} x-u\right)\right\| \\
& \leqq \liminf _{i}\left\|s_{i} x-u\right\| \leqq\|x-u\|,
\end{aligned}
$$

and

$$
s u=w-\lim s_{i} u=u .
$$

For any $G$-invariant closed convex subset $C$ of $K, C$ is also weakly closed, hence $s(C) \subset C$. These imply that $s \in S$ and $S$ is closed in $W$. Now, for any $x \in K$, consider $S x$. Then since for each $s, t \in S$ and $0 \leqq k \leqq 1, k s+(1-k) t \in S$ and $S$ is a semigroup, $S x$ is a $G$-invariant closed convex subset of $K$. Since $B$ is strictly convex, there is a unique point $w \in S x$ such that $\|w-v\|=\min \{\|w-y\|: y \in S x\}$. For any $s \in S, \quad s w \in S x$ and $\|s w-v\| \leqq\|w-v\|$, hence $s w=w$. By Proposition 2 there exists a retraction $r \in S$ of $K$ onto $F(S)=F(G)$. Since $F(r)=F(G), r$ is quasi-nonexpansive.

Corollary 5. Let $K$ be a nonempty bounded closed convex subset of a uniformly convex Banach space, $f$ a continuous generalized nonexpansive mapping of $K$ into $K$. Then there exists a quasinonexpansive retraction $r$ of $K$ onto $F(f)$ such that any $f$-invariant closed convex subset of $K$ is r-invariant.

Proof. By the fixed point theorem of Goebel, Kirk, and Shimi [18] $F(f)$ is nonempty, hence $f$ is quasi-nonexpansive.

REMARK 1. Let $r$ be a quasi-nonexpansive retraction of a subset $K$ of a Banach space into itself, then for any $x, y \in K$, it follows that $\|r x-r y\| \leqq 1 / 2\{\|x-r y\|+\|y-r x\|\}$. This is a special form of generalized nonexpansive mapping. 
If $K$ is a subset of a Banach space and $K$ has normal structure, then the following holds. For the definition of normal structure and related results, see for example Lim [28].

THEOREM 6. Let $K$ be a nonempty weakly compact convex subset of a Banach space, $G$ a left reversible semitopological semigroup acting on $K$. If the action of $G$ on $K$ is separately continuous, nonexpansive and $K$ has normal structure, then there exists a nonexpansive retraction $r$ of $K$ onto $F(G)$ such that every $G$-invariant closed convex subset of $K$ is r-invariant.

Proof. By theorems of Holmes and Lau [21], Lim [28], $F(G)$ is nonempty. Define $S=\{f: K \rightarrow K: f$ is nonexpansive with $F(f) \supset$ $F(G)$ and every $G$-invariant closed convex subset of $K$ is $f$-invariant\}. Then $S$ is a semigroup and compact in the topology of pointwise weak convergence as in the proof of Theorem 5. Moreover, for any $x \in K, S x$ is $G$-invariant and weakly compact, hence there is a common fixed point of $G$ in $S x$. This point is also a fixed point of $S$. Therefore Proposition 2 implies that there exists in $S$ a retraction $r$ of $K$ onto $F(S)=F(G)$.

The following theorem is a common fixed point theorem for a left reversible semigroup of nonexpansive mappings and a multivalued nonexpansive mapping in a Banach space which satisfies Opial's condition. Concerning results related to Opial's condition, we refer the reader to $[20,25]$.

THEOREM 7. Let $K$ be a nonempty weakly compact convex subset of a Banach space which satisfies Opial's condition, $G$ a left reversible semitopological semigroup acting on $K, T$ a nonexpansive mapping of $K$ into $2^{K}$ such that for each $x \in K, T x$ is nonempty compact convex. Suppose the action of $G$ on $K$ is separately continuous and nonexpansive. If $G$ and $T$ commute, then there exists an element $z \in K$ such that $g z=z \in T z$ for all $g \in G$.

Proof. $K$ has normal structure by a theorem of Gossez and Lami Dozo [20]. Hence, by Theorem 6 there is a nonexpansive retraction $r$ of $K$ onto $F(G)$ for which every $G$-invariant closed convex subset of $K$ is $r$-invariant. Define a mapping $S$ of $K$ into $2^{K}$ by $S x=T(r x) \quad(x \in K)$, then $S$ is nonexpansive. Thus, there exists a fixed point $v$ of $S$ in $K$ by Lami Dozo's fixed point theorem [25]. Since $G$ and $T$ commute, $S v$ is $G$-invariant, hence $r v \in S v$. Let $z=r v$. Then it follows that $g z=z \in T z$ for all $g \in G$. 
In a uniformly convex Banach space we can prove the following common fixed point theorem for a family of quasi-nonexpansive mappings and a multivalued nonexpansive mapping.

THEOREM 8. Let $K$ be a nonempty bounded closed convex subset of a uniformly convex Banach space, $G$ a family of mappings of $K$ into $K$ for which $F(G)$ is nonempty and each $g \in G$ is nonexpansive with respect to $F(G), T$ a nonexpansive mapping of $K$ into $2^{K}$, where for any $x \in K, T x$ is nonempty compact convex. If $G$ and $T$ commute weakly, then there exists a point $z \in K$ such that $g z=z \in T z$ for all $g \in G$.

Proof. Let $x \in F(G)$. Then for any $g \in G, g\left(b d_{K} T x\right) \subset T(g x)=T x$ and $g$ is nonexpansive with respect to $F(G)$, hence it follows that $T x \cap F(G) \neq \varnothing$, since a unique point $w \in b d_{K} T x$ nearest to $x$ is a common fixed point of $G$. Define a multivalued mapping $S$ of $F(G)$ into $2^{F(G)}$ by $S x=T x \cap F(G)(x \in F(G))$. Then $S$ is nonexpansive. In fact, for any $x, y \in F(G)$,

$$
\begin{aligned}
D(S x, S y) & =\max \left\{\sup _{u \in S x} d(u, S y), \sup _{v \in S y} d(v, S x)\right\} \\
& =\max \left\{\sup _{u \in S x} d(u, T y), \sup _{v \in S y} d(v, T x)\right\} \\
& \leqq \max \left\{\sup _{u \in T x} d(u, T y), \sup _{v \in T y} d(v, T x)\right\} \\
& =D(T x, T y) \leqq\|x-y\|,
\end{aligned}
$$

where $d(b, A)=\inf \{\|b-a\|: a \in A\}$. Now by Lim's fixed point theorem [29], there exists an element $z \in F(G)$ for which $z \in S z$. Thus we obtain $g z=z \in T z$ for all $g \in G$.

As direct consequences of Theorem 8, we have the following corollaries.

CoROllary 6. Let $K$ be a nonempty bounded closed convex subset of a uniformly convex Banach space, $G$ a left reversible semitopological semigroup acting on $K, T$ a nonexpansive mapping of $K$ into $2^{K}$ such that for each $x \in K$, Tx is nonempty compact convex. Suppose the action of $G$ on $K$ is separately continuous and nonexpansive. If $G$ and $T$ commute weakly, then there is a $z \in K$ such that $g z=z \in T z$ for all $g \in G$.

Proof. Browder [6] showed that $K$ has normal structure, hence $F(G)$ is nonempty by theorems of Holmes and Lau [21] and Lim [28].

COROLlary 7. Let $K$ be a nonempty bounded closed convex sub- 
set of a uniformly convex Banach space, $f$ a continuous generalized nonexpansive mapping of $K$ into $K, T$ a nonexpansive mapping of $K$ into $2^{K}$, where for any $x \in K$, Tx is nonempty compact convex. If $f$ and $T$ commute weakly, then there exists an element $z \in K$ such that $f z=z \in T z$.

Proof. $f$ is quasi-nonexpansive, since $F(f)$ is nonempty by the theorem of Goebel, Kirk, and Shimi [18].

5. Fixed point theorems in Hilbert spaces. Throughout this section let $H$ be a Hilbert space. For the sake of simplicity we assume that $H$ is real. For each nonempty closed convex subset $M$ of $H$, we denote by $P_{M}$ the projection of $H$ onto $M$, and recall that $P_{M}$ is nonexpansive (cf. Phelps [32]).

THEOREM 9. Let $K$ be a nonempty closed convex subset of $H, f$ a continuous quasi-nonexpansive mapping of $K$ into $K$ and $M=F(f)$. Then for each compact convex subset $C$ of $K$ such that $f\left(b d_{K} C\right) \subset C$, we have $P_{M}(C) \subset C$.

Proof. Suppose $C$ is a compact convex subset of $K$ such that $f\left(b d_{K} C\right) \subset C$. Take a sequence $\left\{k_{n}\right\}$ of real numbers for which $0<k_{n}<1$ and $k_{n} \rightarrow 0$ as $n \rightarrow \infty$. Fix an element $w \in C$ and for each $n$, define a mapping $f_{n}$ of $C$ into $K$ by

$$
f_{n} x=k_{n} w+\left(1-k_{n}\right) f x \quad(x \in C),
$$

then since $f_{n}$ is continuous and $C$ is compact, by a theorem of Fan [16] there exists a point $y_{n} \in C$ such that

$$
\left\|f_{n} y_{n}-y_{n}\right\|=\min \left\{\left\|f_{n} y_{n}-x\right\|: x \in C\right\} \text {. }
$$

Since $f\left(b d_{K} C\right) \subset C$ implies $f_{n}\left(b d_{K} C\right) \subset C$, we obtain $f_{n} y_{n}=y_{n}$. We may assume that $\left\{y_{n}\right\}$ converges to some $v \in C$. This $v$ is a fixed point of $f$. In fact, choose any $u \in F(f)$, then by quasi-nonexpansiveness of $f$ we have $\left\|f y_{n}-u\right\| \leqq\left\|y_{n}-u\right\|$. Hence $\left\{f y_{n}\right\}$ is bounded since $C$ is bounded (compact). This implies that $\left\|y_{n}-f y_{n}\right\|=k_{n}\left\|w-f y_{n}\right\| \rightarrow 0$ as $n \rightarrow \infty$. Thus the right hand side of the inequality

$$
\|f v-v\| \leqq\left\|f v-f y_{n}\right\|+\left\|f y_{n}-y_{n}\right\|+\left\|y_{n}-v\right\|
$$

tends to 0 as $n \rightarrow \infty$ and we obtain $f v=v$. Now we show that $P_{M} w=v$ by methods employed by Browder [7]. We have

$$
y_{n}-P_{M} w=\frac{1-k_{n}}{k_{n}}\left(f y_{n}-y_{n}\right)+w-P_{M} w,
$$

hence 


$$
\begin{aligned}
\left\|y_{n}-P_{M} w\right\|^{2}= & \frac{1-k_{n}}{k_{n}}\left(f y_{n}-y_{n}, y_{n}-P_{M} w\right) \\
& +\left(w-P_{M} w, y_{n}-P_{M} w\right)
\end{aligned}
$$

Since $f$ is quasi-nonexpansive, we obtain

$$
\left((I-f) y_{n}-(I-f) P_{M} w, y_{n}-P_{M} w\right) \geqq 0,
$$

where $I$ is the identity mapping on $H$. Thus

$$
\left(f y_{n}-y_{n}, y_{n}-P_{M} w\right) \leqq 0 \text {. }
$$

Also, since $\left(w-P_{M} w, P_{M} w-v\right) \geqq 0$, we have

$$
\left(w-P_{M} w, y_{n}-P_{M} w\right) \leqq\left(w-P_{M} w, y_{n}-v\right) \text {. }
$$

Therefore it follows that

$$
\left\|y_{n}-P_{M} w\right\|^{2} \leqq\left(w-P_{M} w, y_{n}-v\right) .
$$

Since $\left\{y_{n}\right\}$ converges to $v, P_{M} w=v \in C$.

Similarly we have the following

THEOREM 10. Let $K$ be a nonempty closed convex subset of $H$, $f$ a nonexpansive mapping of $K$ into $K$ for which $M=F(f)$ is nonempty. Then for any bounded closed convex subset $C$ of $K$ such that $f\left(b d_{K} C\right) \subset C, P_{M}(C) \subset C$ holds.

Proof. Take a sequence $\left\{k_{n}\right\}$ of real numbers such that $0<k_{n}<1$ and $k_{n} \rightarrow 0$ as $n \rightarrow \infty$. Fix $w \in C$ and for any $n$, define a mapping $f_{n}$ of $C$ into $K$ by

$$
f_{n} x=k_{n} w+\left(1-k_{n}\right) f x \quad(x \in C),
$$

then, since $f_{n}$ is $\left(1-k_{n}\right)$-contraction and $P_{C}$ is nonexpansive, $P_{C} f_{n}$ is a $\left(1-k_{n}\right)$-contraction mapping of $C$ into $C$. Hence there exists a unique fixed point $y_{n} \in C$ of $P_{C} f_{n}$, that is,

$$
\left\|f_{n} y_{n}-y_{n}\right\|=\min \left\{\left\|f_{n} y_{n}-x\right\|: x \in C\right\} .
$$

Since $f\left(b d_{K} C\right) \subset C$ implies $f_{n}\left(b d_{K} C\right) \subset C$, we have $f_{n} y_{n}=y_{n}$. We may assume that $\left\{y_{n}\right\}$ converges weakly to some $v \in C$. The rest of the proof proceeds as in the proof of Theorem 9 by using methods in Browder's paper [7]. In conclusion, we obtain that $f v=v$ by demiclosedness of $I-f$ (cf. [12, Remark 3]) and $P_{M} w=v$.

Proposition 3. Let $K$ be a nonempty bounded closed convex subset of $H, f$ a nonexpansive mapping of $K$ into $H$. Then there exists 
a point $z \in K$ such that $\|f z-z\|=\min \{\|f z-x\|: x \in K\}$.

Proof. Since $P_{M} f$ is a nonexpansive mapping of $K$ into $K$, by Browder's fixed point theorem [5] there exists a $z \in K$ such that $P_{K} f z=z$. For this $z$, we have the desired equality.

REMark 2. We do not know whether Proposition 3 is true when $K$ is only required to be weakly compact convex and to have normal structure in a Banach space.

Now we can prove a common fixed point theorem for a quasinonexpansive mapping and a multivalued nonexpansive mapping in a Hilbert space.

THEOREM 11. Let $K$ be a nonempty bounded closed convex subset of $H, f$ a continuous mapping of $K$ into $H, T$ a nonexpansive mapping of $K$ into $2^{K}$, where for any $x \in K$, Tx is nonempty compact convex. Suppose $M=\{x \in K:\|f x-x\|=\min \{\|f x-y\|: y \in K\}\}$ is nonempty and $f$ is nonexpansive with respect to $M$. If for each $x \in K, P_{K} f\left(b d_{K} T x\right) \subset T\left(P_{K} f x\right)$, then there exists an element $z \in K$ such that $\left\|f_{z}-z\right\|=\min \{\|f z-x\|: x \in K\}$ and $z \in T z$.

Proof. It is obvious that $M=F\left(P_{K} f\right)$. For any $x \in K, u \in M$, we have

$$
\begin{aligned}
\left\|P_{K} f x-u\right\| & =\left\|P_{K} f x-P_{K} f u\right\| \\
& \leqq\|f x-f u\| \leqq\|x-u\| .
\end{aligned}
$$

Hence $P_{K} f$ is a quasi-nonexpansive mapping of $K$ into $K$. Define a mapping $S$ of $K$ into $2^{K}$ by $S x=T\left(P_{M} x\right)(x \in K)$. Then $S$ is nonexpansive and has a fixed point $v$ in $K$. Since

$$
\begin{aligned}
P_{K} f\left(b d_{K} S v\right) & =P_{K} f\left(b d_{K} T\left(P_{M} v\right)\right) \\
& \subset T\left(P_{K} f\left(P_{M} v\right)\right)=T\left(P_{M} v\right)=S v,
\end{aligned}
$$

by Theorem 9 it follows that $P_{K}(S v) \subset S v$. In particular $P_{M} v \in S v$. Denote $z=P_{k} v$. Then we have $P_{K} f z=z \in T z$.

REMARK 3. Theorem 11 is also a corollary to Theorem 8, but the proof given above is a constructive one. Compare this with the proofs of Theorem 3 and Theorem 7.

\section{REFERENCES}

1. N. A. Assad and W. A. Kirk, Fixed point theorems for set-valued mappings of contractive type, Pacific J. Math., 43 (1972), 553-562. 
2. L. P. Belluce and W. A. Kirk, Fixed-point theorems for families of contraction mappings, Pacific J. Math., 18 (1966), 213-217.

3. L. P. Belluce and W. A. Kirk, Nonexpansive mappings and fixed-points in Banach spaces, Illinois J. Math., 11 (1967), 474-479.

4. H. F. Bohnenblust and S. Karlin, On a theorem of Ville, in "Contributions to the Theory of Games I" (H. W. Kuhn and A. W. Tucker, Ed.), 155-160, Princeton University Press, Princeton, New Jersey, 1950.

5. F. E. Browder, Fixed-point theorems for noncompact mappings in Hilbert space, Proc. Nat. Acad. Sci. U.S.A., 53 (1965), 1272-1276.

6. - Nonexpansive nonlinear operators in a Banach space, Proc. Nat. Acad. Sci. U.S.A., 54 (1965), 1041-1044.

7. - Convergence of approximants to fixed points of nonexpansive nonlinear mappings in Banach spaces, Arch. Rational Mech. Anal., 24 (1967), 82-90.

8. - The fixed point theory of multi-valued mappings in topological vector spaces, Math. Ann., 177 (1968), 283-301.

9. R. E. Bruck, Jr., Properties of fixed-point sets of nonexpansive mappings in Banach spaces, Trans. Amer. Math. Soc., 179 (1973), 251-262.

10. - A common fixed-point theorem for a commuting family of nonexpansive mappings, Pacific J. Math., 53 (1974), 59-71.

11. R. E. De Marr. Common fixed points for commuting contraction mappings, Pacific J. Math., 13 (1963), 1139-1141.

12. W. G. Dotson, Jr, Fixed point theorems for non-expansive mappings on star-shaped subsets of Banach spaces, J. London Math. Soc., (2) 4 (1972), 408-410.

13. - Fixed points of quasi-nonexpansive mappings, J. Austral. Math. Soc., 13 (1972), 167-170.

14. M. Edelstein, On non-expansive mappings of Banach spaces, Proc. Cambridge Philos. Soc., 60 (1964), 439-447.

15. K. Fan, Fixed-point and minimax theorems in locally convex topological linear spaces, Proc. Nat. Acad. Sci. U. S. A., 38 (1952), 121-126.

16. Extensions of two fixed point theorems of F. E. Browder, Math. Z., 117 (1969), 234-240.

17. I. L. Glicksberg, A further generalization of the Kakutani fixed point theorem with applications to Nash equilibrium points, Proc. Amer. Math. Soc., 3 (1952), 170-174.

18. K. Goebel, W. A. Kirk, and T. N. Shimi, A fixed point theorem in uniformly convex spaces, Boll. Un. Mat. Ital., (4) 7 (1973), 67-75.

19. D. Göhde, Zum Prinzip der kontraktiven Abbildung, Math. Nachr., 30 (1965), 251-258.

20. J.-P. Gossez and E. Lami Dozo, Some geometric properties related to the fixed point theory of nonexpansive mappings, Pacific J. Math., 40 (1972), 563-573.

21. R. D. Holmes and A. T. Lau, Nonexpansive actions of topological semigroups and fixed points, J. London Math. Soc., (2) 5 (1972), 563-573.

22. S. Itoh and W. Takahashi, Single-valued mappings, multivalued mappings and fixed-point theorems, J. Math. Anal. Appl., 59 (1977), 514-521.

23. W. A. Kirk, A fixed point theorem for mappings which do not increase distances, Amer. Math. Monthly, 72 (1965), 1004-1006.

24. - Fixed point theorems for nonlinear nonexpansive and generalized contraction mappings, Pacific J. Math., 38 (1971), 89-94.

25. E. Lami Dozo, Multivalued nonexpansive mappings and Opial's condition, Proc. Amer. Math. Soc., 38 (1973), 286-292.

26. A. T. Lau, Invariant means on almost periodic functions and fixed point properties, Rockey Mountain J. Math., 3 (1973), 69-76.

27. T.-C. Lim, A fixed point theorem for families of nonexpansive mappings, Pacific J. Math., 53 (1974), 487-493. 
28. T.-C. Lim, Characterizations of normal structure, Proc. Amer. Math. Soc., 43 (1974), 313-319.

29. T.-C. Lim, A fixed point theorem for multivalued nonexpansive mappings in a uniformly convex Banach space, Bull. Amer. Math. Soc., 80 (1974), 1123-1126.

30. J. T. Markin, A fixed point theorem for set valued mappings, Bull. Amer. Math. Soc., 74 (1968), 639-640.

31. T. Mitchell, Fixed points of reversible semigroups of nonexpansive mappings, Kōdai Math. Sem. Rep., 22 (1970), 322-323.

32. R. R. Phelps, Convex sets and nearest points, Proc. Amer. Math. Soc., 8 (1957), 790-797.

33. W. Takahashi, Fixed point theorem for amenable semigroups of nonexpansive mappings, Kōdai Math. Sem. Rep., 21 (1969), 383-386.

34. - On fixed points for reversible semigroups of nonexpansive mappings, Sci. Rep. Yokohama Nat. Univ., 19 (1972), 1-4.

35. - Nonlinear variational inequalities and fixed point theorems, J. Math. Soc. Japan, 28 (1976), 168-181.

Received March 3, 1978 and in revised form May 15, 1978.

Department of Information Sciences

TOKYo Institute of TECHNOLOGY

OH-OKayama, Meguro-KU, TOKYo 152, JaPAN 


\section{PACIFIC JOURNAL OF MATHEMATICS}

\section{EDITORS}

RichaRd ARENS (Managing Editor)

University of California

Los Angeles, CA 90024

Charles W. Curtis

University of Oregon

Eugene, OR 97403

C. C. Moore

University of California

Berkeley, CA 94720

\section{J. DUGUNDJI}

Department of Mathematics

University of Southern California

Los Angeles, CA 90007

R. FinN and J. Milgram

Stanford University

Stanford, CA 94305

\section{E. F. BECKENBACH \\ B. H. NeumanN

\author{
UNIVERSITY OF BRITISH COLUMBIA \\ CALIFORNIA INSTITUTE OF TECHNOLOGY \\ UNIVERSITY OF CALIFORNIA \\ MONTANA STATE UNIVERSITY \\ UNIVERSITY OF NEVADA, RENO \\ NEW MEXICO STATE UNIVERSITY \\ OREGON STATE UNIVERSITY \\ UNIVERSITY OF OREGON
}

F. WOLF

K. Yoshida

\section{SUPPORTING INSTITUTIONS}

\author{
UNIVERSITY OF SOUTHERN CALIFORNIA \\ STANFORD UNIVERSITY \\ UNIVERSITY OF HAWAII \\ UNIVERSITY OF TOKYO \\ UNIVERSITY OF UTAH \\ WASHINGTON STATE UNIVERSITY \\ UNIVERSITY OF WASHINGTON
}

The Supporting Institutions listed above contribute to the cost of publication of this Journal, but they are not owners or publishers and have no responsibility for its content or policies.

Mathematical papers intended for publication in the Pacific Journal of Mathematics should be in typed form or offset-reproduced, (not dittoed), double spaced with large margins. Please do not use built up fractions in the text of the manuscript. However, you may use them in the displayed equations. Underline Greek letters in red, German in green, and script in blue. The first paragraph or two must be capable of being used separately as a synopsis of the entire paper. Items of the bibliography should not be cited there unless absolutely necessary, in which case they must be identified by author and journal, rather than by item number. Manuscripts, in triplicate, may be sent to any one of the editors. Please classify according to the scheme of Math. Reviews, Index to Vol. 39. All other communications should be addressed to the managing editor, or Elaine Barth, University of California, Los Angeles, California, 90024.

50 reprints to each author are provided free for each article, only if page charges have been substantially paid. Additional copies may be obtained at cost in multiples of 50 .

The Pacific Journal of Mathematics is issued monthly as of January 1966. Regular subscription rate: $\$ 72.00$ a year (6 Vols., 12 issues). Special rate: $\$ 36.00$ a year to individual members of supporting institutions.

Subscriptions, orders for numbers issued in the last three calendar years, and changes of address should be sent to Pacific Journal of Mathematics, P.O. Box 969, Carmel Valley, CA 93924, U.S.A. Older back numbers obtainable from Kraus Periodicals Co., Route 100, Millwood, NY 10546.

PUBLISHED BY PACIFIC JOURNAL OF MATHEMATICS, A NON-PROFIT CORPORATION

Printed at Kokusai Bunken Insatsusha (International Academic Printing Co., Ltd.). 8-8, 3-chome, Takadanobaba, Shinjuku-ku, Tokyo 160, Japan. 


\section{Pacific Journal of Mathematics \\ Vol. 79, No. $2 \quad$ June, 1978}

David R. Adams, Quasi-additivity and sets of finite $L^{p}$-capacity ........ 283

George M. Bergman and Warren Dicks, Universal derivations and universal

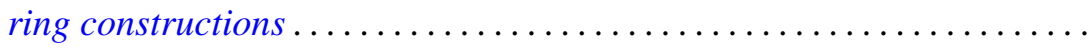

Robert F. Brown, Addendum to: "Fixed points of automorphisms of compact Lie groups".........................................

Eugene Frank Cornelius, Jr., Characterization of a class of torsion free

groups in terms of endomorphisms .......................

Andres del Junco, A simple measure-preserving transformation with trivial centralizer..................................... 357

Allan Lee Edmonds, Extending a branched covering over a handle ...... 363

Sjur Flam, A characterizaton of $\mathbf{R}^{2}$ by the concept of mild convexity .......

Claus Gerhardt, $L^{p}$-estimates for solutions to the instationary Navier-Stokes

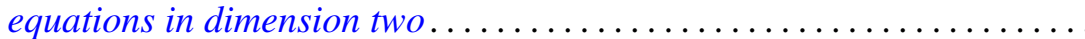

Kensaku Gomi, Finite groups with a standard subgroup isomorphic to

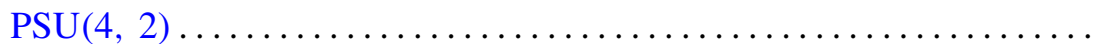

E. E. Guerin, A convolution related to Golomb's root function ........... 463

$\mathrm{H}$. B. Hamilton, Modularity of the congruence lattice of a commutative cancellative semigroup .................................

Stephen J. Haris, Complete reducibility of admissible representations over function fields.

Shigeru Itoh and Wataru Takahashi, The common fixed point theory of singlevalued mappings and multivalued mappings... ...

James E. Joseph, Multifunctions and graphs .............

Bruce Magurn, Images of $S K_{1} Z G$. .

Arnold Koster Pizer, A note on a conjecture of Hecke... .

Marlon C. Rayburn, Maps and h-normal spaces ...........

Barada K. Ray and Billy E. Rhoades, Corrections to: "Fixed-point theorems for mappings with a contractive iterate" ..............

Charles Irvin Vinsonhaler, Corrections to: "Torsion free abelian groups quasiprojective over their endomorphism rings. II". . . 